\title{
Effect of Material Thickness on Attenuation (dB) of PTFE Using Finite Element Method at X-Band Frequency
}

\author{
Abubakar Yakubu, ${ }^{1}$ Zulkifly Abbas, ${ }^{1}$ and Mansor Hashim ${ }^{2}$ \\ ${ }^{1}$ Department of Physics, Universiti Putra Malaysia (UPM), 43400 Serdang, Selangor, Malaysia \\ ${ }^{2}$ Institute of Advance Technology (ITMA), Universiti Putra Malaysia (UPM), 43400 Serdang, Malaysia \\ Correspondence should be addressed to Abubakar Yakubu; abulect73@yahoo.com
}

Received 4 February 2014; Accepted 12 March 2014; Published 3 April 2014

Academic Editor: Qi-Long Zhang

Copyright (c) 2014 Abubakar Yakubu et al. This is an open access article distributed under the Creative Commons Attribution License, which permits unrestricted use, distribution, and reproduction in any medium, provided the original work is properly cited.

PTFE samples were prepared with different thicknesses. Their electric field intensity and distribution of the PTFE samples placed inside a rectangular waveguide were simulated using finite element method. The calculation of transmission/reflection coefficients for all samples thickness was achieved via FEM. Amongst other observable features, result from calculation using FEM showed that the attenuation for the $15 \mathrm{~mm}$ PTFE sample is $-3.32 \mathrm{~dB}$; the $30 \mathrm{~mm}$ thick PTFE sample has an attenuation of $0.64 \mathrm{~dB}$, while the $50 \mathrm{~mm}$ thick PTFE sample has an attenuation of $1.97 \mathrm{~dB}$. It then suffices to say that, as the thickness of the PTFE sample increases, the attenuation of the samples at the corresponding thicknesses increases.

\section{Introduction}

Knowledge of materials behaviour placed in an electromagnetic field is of immense importance especially when it relates to military hardware, electronics, communication, and industrial applications. The measurement of $T / R$ coefficient of materials in the microwave frequency range is found in numerous areas. A good understanding of $T / R$ measurement of these materials and its attenuation is necessary to get useful information from materials proposed for use in microwave absorption.

Over the years, numerous methods have been used to calculate the $T / R$ coefficient of samples at microwave frequency. In the recent, Dudek et al. (1992) and Kumar et al. (2007), the vector network analyzer (VNA) has been used successfully to obtain the $S$ parameters of samples in the microwave wave range $[1,2]$.

A new approach is presented that relies upon 3D electromagnetic simulation results to characterize and calculate the $T / R$ coefficient using FEM. The COMSOL software [3] is based on finite element method (FEM) [3, 4] and this method has been used to simulate rectangular waveguide with three dimensions $[5,6]$. The $3 \mathrm{D}$ simulation results may be used to replace the need for complex theoretical analysis of the measurement geometry. The method is applied to an Xband rectangular waveguide setup, for which the theoretical $S$-parameters can be readily calculated. A PTFE sample is used in our work for all measurements and calculations. Results obtained from simulations are then compared to find the best thickness in terms of attenuation of signal at X-band frequency.

\section{Theory and Methodology}

The analysis in FEM involves four important steps [7]. These steps include (a) discretizing the solution region into finite number of elements, (b) deriving governing equations for a typical element, (c) assembling of all elements in the solution region, and (d) solving the system of equations obtained. It is considered that the electric field is uniform within an element. Thus,

$$
E_{e}=-\nabla V_{e}=-\left(b a_{x}+c a_{y}\right) .
$$

The elements in a solution region can be calculated using the following equation:

$$
A=\frac{1}{2}\left[\left(x_{2}-x_{1}\right)\left(y_{3}-y_{1}\right)-\left(x_{3}-x_{1}\right)\left(y_{2}-y_{1}\right)\right],
$$


where $A$ is the area of the elements $e$ in a domain. After establishing the governing equations the next step is to assemble all elements in the solution region. The energy dissipated within the assembling of all elements in the mesh is given as

$$
w=\sum_{e=1}^{N} W_{e}=\frac{1}{2} \varepsilon[V]^{T}[C][V]
$$

where $n$ is the number of nodes, $N$ is the number of elements, $[C]$ is the overall global coefficient matrix, and $[V]$ is the potential.

In the methodology, we need to calculate the values of $S_{11}$ and $S_{21}$ using the FEM. In this method, the wave equation of a wave guide is paramount in calculating the $S$ magnitudes. The equation of a wave guide is given as [8-10]

$$
\nabla x\left(\mu_{r}^{-1} \nabla x E_{z}\right)-\left(\varepsilon_{r}-j \frac{\sigma}{w \varepsilon_{0}}\right) k_{0}^{2} E_{z}=0 .
$$

In (4), $\mu_{r}$ is relative permeability, $k_{0}$ is free pace wave number, $j$ is imaginary unit, $\sigma$ is conductivity, $w$ is angular frequency, $\varepsilon_{r}$ is relative permittivity, and $\varepsilon_{0}$ is permittivity of air. The vector wave equation for transverse fields can be obtained if there is no conduction current. Thus, the wave equation is given as [11]

$$
\begin{gathered}
\nabla_{t}^{2} E_{t}+\nabla_{t}\left[E_{t} \cdot \nabla_{t} \ln \varepsilon^{*}(x, y)\right]+\left[k_{0}^{2} \varepsilon^{*}(x, y)+\gamma^{2}\right] E_{t}=0 \\
\nabla_{t}^{2} E_{t}+\left[\nabla_{t} \ln \varepsilon^{*}(x, y)\right] \times\left(\nabla_{t} \times H_{t}\right) \\
+\left[k_{0}^{2} \varepsilon^{*}(x, y)+\gamma^{2}\right] H_{t}=0
\end{gathered}
$$

where $k_{0}=2 \pi / \lambda_{0}$ is the free space wave number, $\gamma$ is the propagation constant, $\varepsilon^{*}(x, y)$ is the complex permittivity profile, and $\nabla_{t}$ is the vector Laplacian operator.

In the FEM, first of all we assign our work in the $3 \mathrm{D}$ work plane before going into the RF module, where electromagnetic wave is selected for harmonic waves. The length of the wave guide is $20 \mathrm{~cm}$ long and this was drawn within the work plane, while the length and width of the port of the wave guide was drawn $(2.228 \mathrm{~cm}$ by $1.143 \mathrm{~cm})$. Axis grids, parameter constants, cut-off frequency of simulation, and frequency range are all assigned. It is important to note that the model consists of two rectangular waveguides with microwave excitation along them. The transition mode through the rectangular waveguide used in our work is the $\mathrm{TE}_{10}$ mode. The complex permittivity value used in the simulation is $(2.03-j * 0.002)$ and the permeability value used in our simulation is 1 [12].

Attenuation is a function which can be affected by some factors. One factor affecting attenuation that would be discussed in this work is sample thickness. Thickness of material has influence on the attenuation coefficients of materials [13]. Attenuation of PTFE at thicknesses of $15 \mathrm{~mm}$, $30 \mathrm{~mm}$, and $50 \mathrm{~mm}$ is calculated in this work. The formula
TABLE 1: Corresponding $S$ parameter values with material thickness.

\begin{tabular}{lc}
\hline Thickness $(\mathrm{mm})$ & $S_{21}$ \\
\hline 15 & 0.939 \\
30 & 0.935 \\
50 & 0.926 \\
\hline
\end{tabular}

used to calculate attenuation for calculated FEM result is given as [14]

Attenuation $(\mathrm{dB})$

$$
=-20 \log _{10}\left(\frac{\text { maximum transmitted intensity }}{\text { maximum incident intensity }}\right) \text {. }
$$

\section{Result and Discussion}

The FEM calculated results for $S_{11}$ and $S_{21}$ for the $15 \mathrm{~mm}$, $30 \mathrm{~mm}$, and $50 \mathrm{~mm}$ thick PTFE samples using FEM are shown in Figure 1.

The result obtained from simulation of the PTFE sample for different thicknesses showed that the transmission coefficient for all thicknesses was greater than the reflection coefficient. For the $15 \mathrm{~mm}$ thick PTFE sample, the mean transmission coefficients were calculated to be equal to 0.939 and the reflection coefficient calculated value was 0.309 . For the $30 \mathrm{~mm}$ thick sample, the calculated transmission coefficient was 0.935 , while the reflection coefficient was 0.284. For the $50 \mathrm{~mm}$ thick PTFE sample, the calculated transmission coefficient was calculated to be 0.926 , while the reflection coefficient was calculated to be 0.343 .

The above analysis confirms that the transmission coefficient depends on the thickness of the sample. The result shows that as the sample thickness increases, the transmission coefficient decreases. The result for $S_{21}$ and material thickness is shown in Table 1 and represented with a graph in Figure 2.

The simulation and determination of the electric field intensity were done using FEM. The result obtained from the simulation is presented in Figure 3, for the $15 \mathrm{~mm}, 30 \mathrm{~mm}$, and $50 \mathrm{~mm}$ PTFE samples, respectively.

The rectangular waveguide that is simulated is divided into three regions as the architecture depicts. These regions are region I, region II, and region III. For this work, regions I and III are encapsulated in air, while region II represents the samples. In the geometry of simulation, the $x$-axis represents the length of the rectangular wave guide encompassing all the three regions.

To determine the intensity in each region of the rectangular waveguide, a plot for the whole rectangular wave guide against amplitude of intensity is extrapolated and presented in Figure 3.

For the calculation of attenuation from the field intensity, two variables are important for the calculation. The two important variables are the highest incident intensity in region I and the highest emergent intensity in region III.

For the $15 \mathrm{~mm}$ thick PTFE sample in Figure 3(a), the highest incident electric intensity at region $I$ is $2,635.465 \mathrm{v} / \mathrm{m}$, while at region III the maximum value of the transmitted 


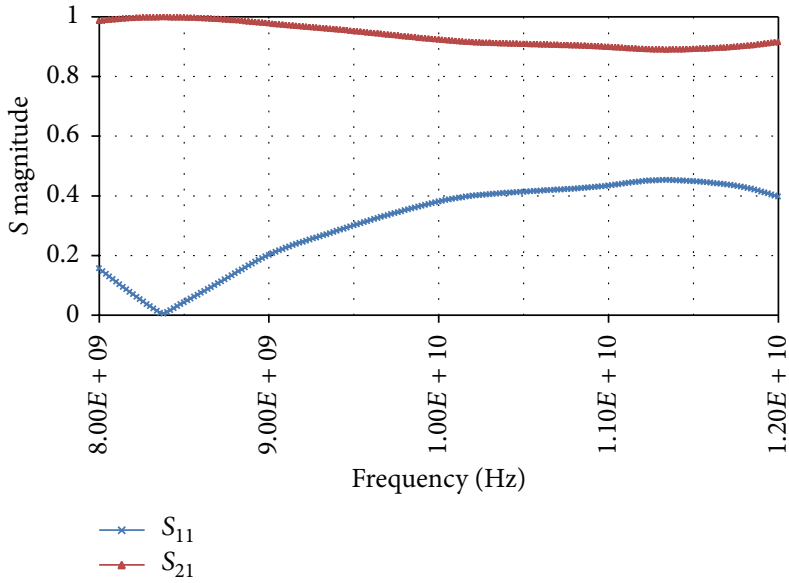

(a)

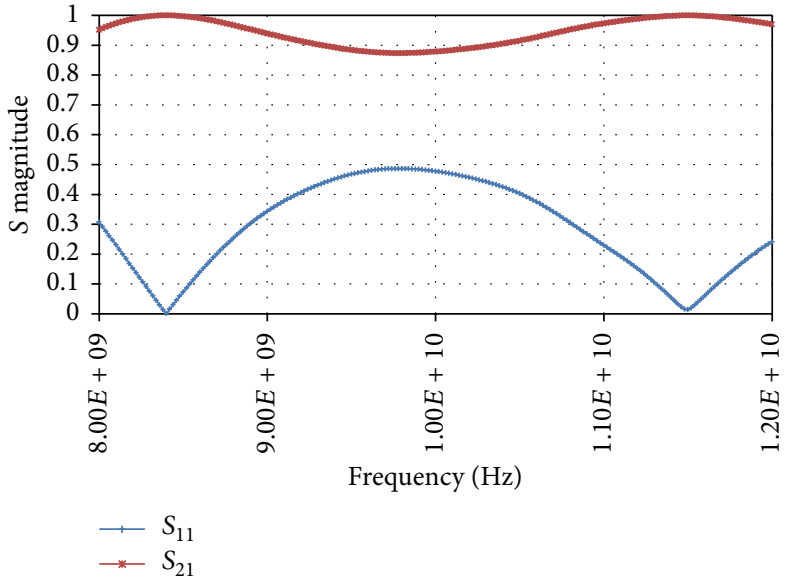

(b)

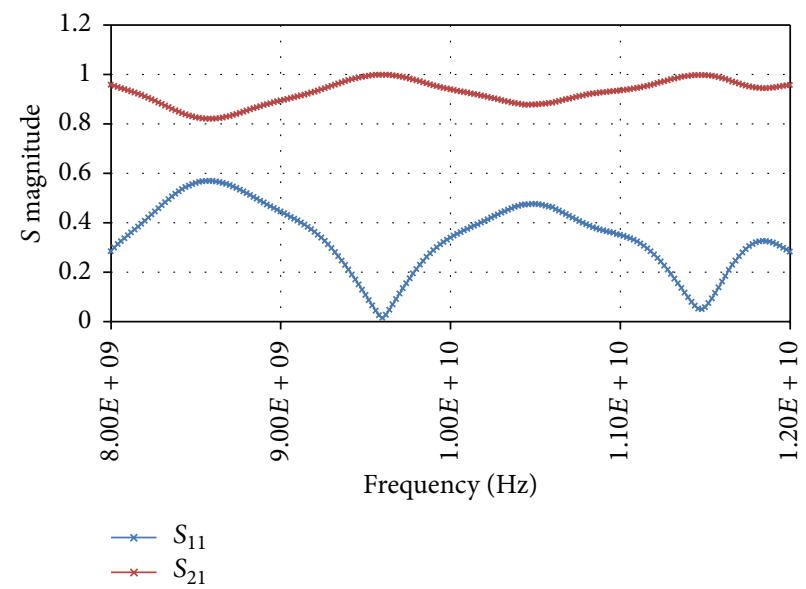

(c)

FIgURE 1: FEM simulated magnitude of $S_{11}$ and $S_{21}$ for $15 \mathrm{~mm}, 30 \mathrm{~mm}$, and $50 \mathrm{~mm}$ PTFE samples, respectively.

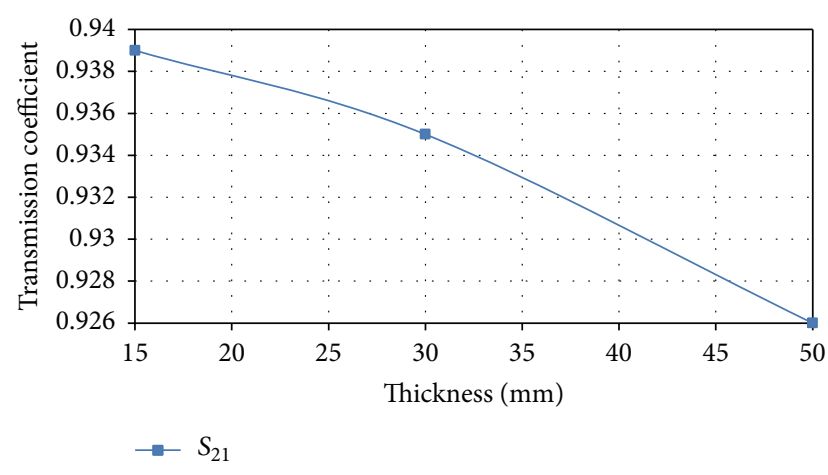

FIGURE 2: Transmission coefficient versus material thickness (PTFE).

intensity is $3,860.375 \mathrm{v} / \mathrm{m}$. Thus from (6), the attenuation for the $5 \mathrm{~mm}$ thick PTFE sample at $12 \mathrm{GHz}$ is calculated to be $-3.32 \mathrm{~dB}$.

For the $30 \mathrm{~mm}$ thick PTFE sample in Figure 3(b), the highest incident electric intensity at region $\mathrm{I}$ is $2856.94 \mathrm{v} / \mathrm{m}$,
TABLE 2: Values of attenuation at different material thicknesses.

\begin{tabular}{lc}
\hline Thickness $(\mathrm{mm})$ & Attenuation $(\mathrm{dB})$ \\
\hline 15 & -3.32 \\
30 & 0.64 \\
50 & 1.97 \\
\hline
\end{tabular}

while at region III the maximum value of transmitted intensity is $3074.13 \mathrm{v} / \mathrm{m}$. Thus from (6), the attenuation for the $30 \mathrm{~mm}$ thick PTFE sample at $12 \mathrm{GHz}$ is calculated to be $0.64 \mathrm{~dB}$.

For the $50 \mathrm{~mm}$ thick PTFE sample in Figure 3(c), the highest incident electric intensity at region $\mathrm{I}$ is $256.36 \mathrm{v} / \mathrm{m}$, while at region III the maximum value of the transmitted intensity is $1.97 \mathrm{v} / \mathrm{m}$. Thus from (6), the attenuation for the $30 \mathrm{~mm}$ thick PTFE sample at $12 \mathrm{GHz}$ is calculated to be $1.97 \mathrm{~dB}$.

Table 2 shows the attenuation of PTFE samples at thicknesses of $15 \mathrm{~mm}, 30 \mathrm{~mm}$, and $50 \mathrm{~mm}$. The result presented in Table 1 is used to plot a graph of attenuation against thickness of materials. Result of plot is shown in Figure 4. 


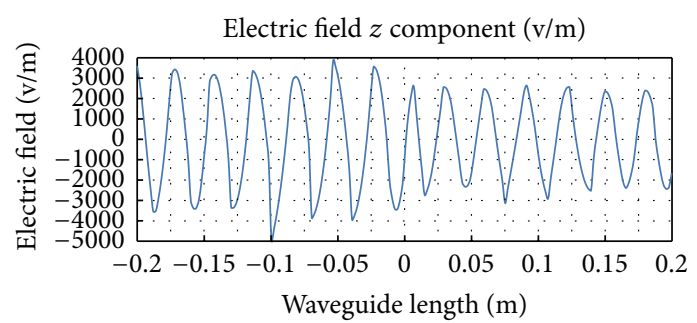

(a)

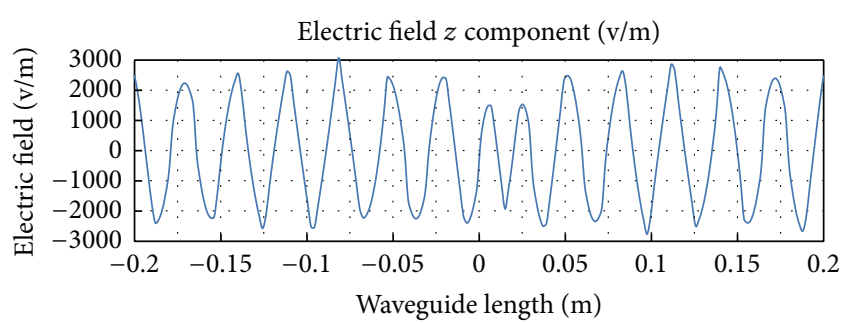

(b)

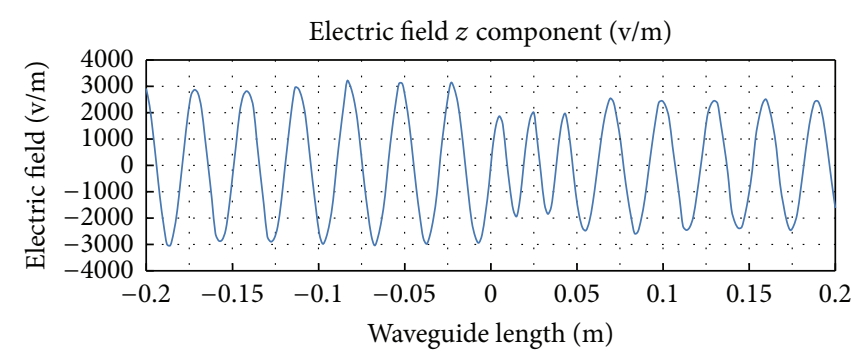

(c)

Figure 3: Electric field intensity for the $15 \mathrm{~mm}, 30 \mathrm{~mm}$, and $50 \mathrm{~mm}$ thick PTFE samples at $12 \mathrm{GHz}$.

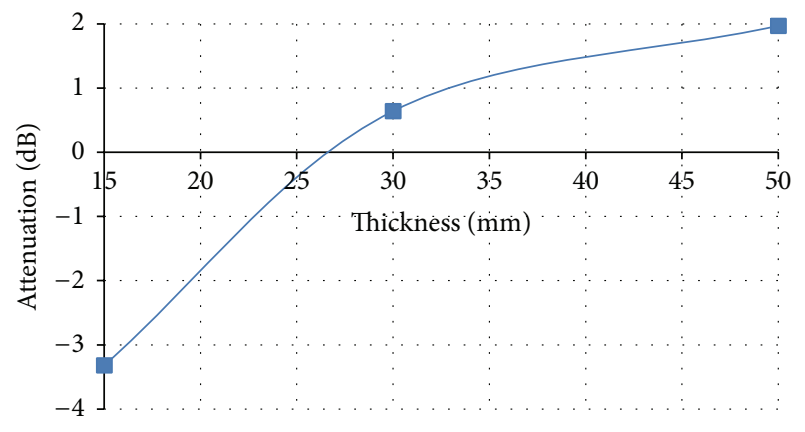

Figure 4: Attenuation versus sample thickness.

Result from the graph reveals that the attenuation for the $15 \mathrm{~mm}$ PTFE sample is $-3.32 \mathrm{~dB}$; the $30 \mathrm{~mm}$ thick PTFE sample has an attenuation of $0.64 \mathrm{~dB}$, while the $50 \mathrm{~mm}$ thick PTFE sample has an attenuation of $1.97 \mathrm{~dB}$. The result shows a sequential behavioural pattern in terms of magnitude of attenuation at these thicknesses. It then implies to say that as the thickness of the PTFE sample increases, the attenuation of the samples at the corresponding thicknesses increases.

\section{Conclusion}

In this work the simulation of electric field intensity, calculation of transmission/reflection coefficients, and determination of attenuation of PTFE sample with different thicknesses were undertaken using FEM. Results obtained for the calculation of the transmission/reflection coefficients revealed that transmission coefficients for all samples were greater in magnitude than values obtained for reflection coefficients. The calculated value for $S_{21}$ using FEM for the $15 \mathrm{~mm}$ thick PTFE sample was 0.939 with a $S_{11}$ value of 0.309 .
The $30 \mathrm{~mm}$ thick PTFE sample had a $S_{21}$ value of 0.935 with a corresponding $S_{11}$ value of 0.284 . Finally, the $50 \mathrm{~mm}$ thick PTFE had a calculated $S_{21}$ value of 0.926 with a corresponding $S_{11}$ value of 0.343 . The $S$ parameter analysis confirmed that the FEM can be used to calculate $S$ magnitude of materials placed in waveguide and the result also confirmed that $S_{21}$ magnitude is greater than $S_{11}$ magnitude with a decreasing trend $S_{21}$ as the sample thickness increases.

The attenuation of the different thicknesses showed that the thicker the sample thickness is, the higher the attenuation of the PTFE sample is.

\section{Conflict of Interests}

The authors declare that there is no conflict of interests regarding the publication of this paper.

\section{Acknowledgment}

The authors wish to thank the Universiti Putra Malaysia (UPM) for its support and for the provision of enabling environment to carry out this work.

\section{References}

[1] K. E. Dudeck and L. J. Buckley, "Dielectric material measurement of thin samples at millimeter wavelengths," IEEE Transactions on Instrumentation and Measurement, vol. 41, no. 5, pp. 723-725, 1992.

[2] A. Kumar, S. Sharma, and G. Singh, "Measurement of dielectric constant and loss factor of the dielectric material at microwave frequencies," Progress in Electromagnetics Research, vol. 69, pp. 47-54, 2007.

[3] The Comsol Multiphysics, "Physics Builder User's Guide," Version 4.3 BETA, Comsol AB, 2012. 
[4] A. L. de Paula, M. C. Rezende, and J. J. Barroso, "Experimental measurements and numerical simulation of permittivity and permeability of teflon in $\mathrm{x}$ band," Journal of Aerospace Technology and Management, vol. 3, no. 1, pp. 59-64, 2011.

[5] H. Soleimani, Z. Abbas, K. Khalid, N. Yahya, and H. Soleimani, "Determination of reflection and transmission coefficient of PTFE at X-band frequency using NRW and FEM methods," Solid State Science and Technology, vol. 17, no. 2, pp. 32-42, 2009.

[6] Hewlett Packard Company, "HP 8719A, HP 8720B, Microwave Network," Analyzer Operating Manual, 1990.

[7] N. O. Sadiku, Elements of Electromagnetic, Oxford University Press, 3rd edition, 1994.

[8] Z. Abbas, R. D. Pollard, and R. W. Kelsall, "Complex permittivity measurements of Ka-band using rectangular dielectric waveguide," IEEE Transactions on Instrumentation and Measurement, vol. 50, no. 5, pp. 1334-1342, 2001.

[9] J. Baker-Jarvis, E. J. Vanzura, and W. A. Kissick, "Improved technique for determining complex permittivity with the transmission/reflection method," IEEE Transactions on Microwave Theory and Techniques, vol. 38, no. 8, pp. 1096-1103, 1990.

[10] J. Krupka, J. Breeze, A. Centeno, N. Alford, T. Claussen, and L. Jensen, "Measurements of permittivity, dielectric loss tangent, and resistivity of float-zone silicon at microwave frequencies," IEEE Transactions on Microwave Theory and Techniques, vol. 54, no. 11, pp. 3995-4000, 2006.

[11] H. Soleimani, Electomagnetic characterisation of yttrium iron garnet and lanthanum iron garnet filled polymer nanocomposites using FEM simulation rectangular waveguide and NRW methods [Ph.D. thesis], Universiti Putra, Serdang, Malaysia, 2009.

[12] Rohde and Schwarz, "Measurement of Dielectric material properties," Application Note, 2012.

[13] M. A. Abdel-Rahman, E. A. Badawi, Y. L. Abdel-Hady, and N. Kamel, "Effect of sample thickness on the measured mass attenuation coefficients of some compounds and elements for 59.54, 661.6 and $1332.5 \mathrm{keV} \gamma$-rays," Nuclear Instruments and Methods in Physics Research, Section A, vol. 447, no. 3, pp. 432436, 2000.

[14] M. A. Ramadan, Electromagnetic characterisation of Sm-YIG and Sm-YIG-PVDF composites prepared using modified conventional mixing oxide technique [Ph.D. thesis], Universiti Putra, Serdang, Malaysia, 2009. 

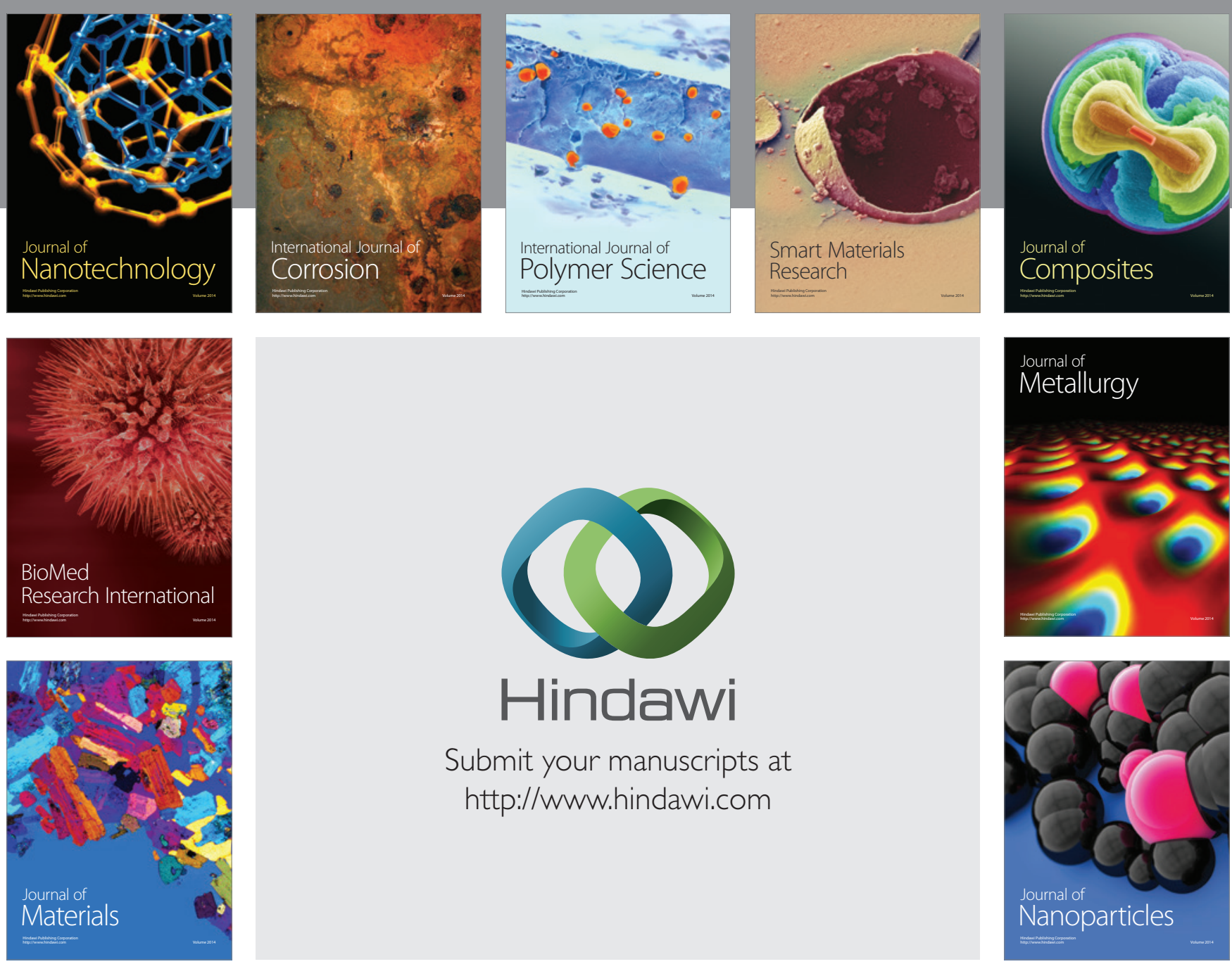

Submit your manuscripts at http://www.hindawi.com
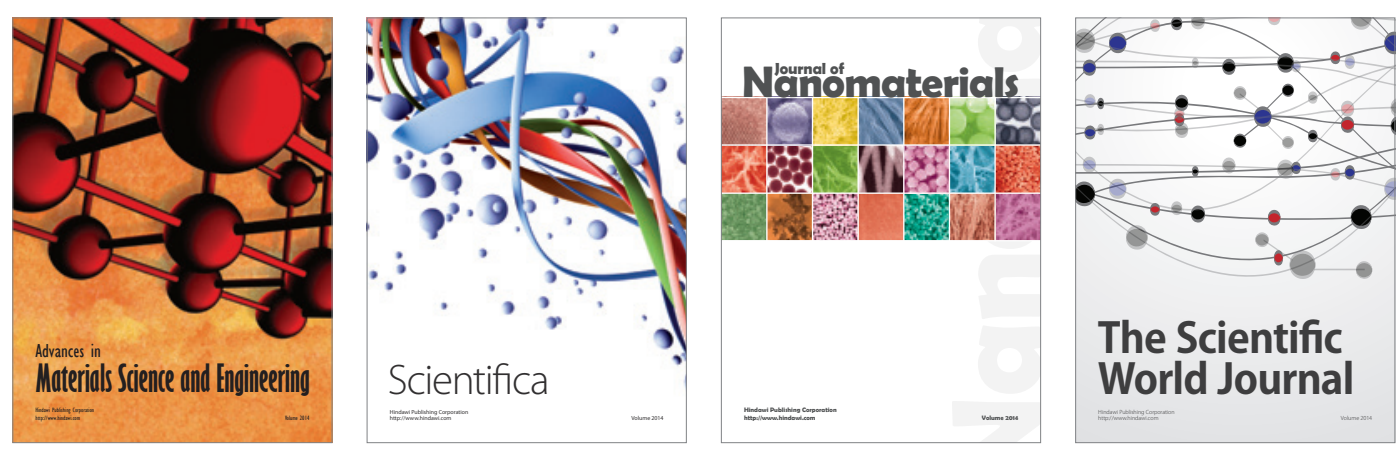

\section{The Scientific World Journal}
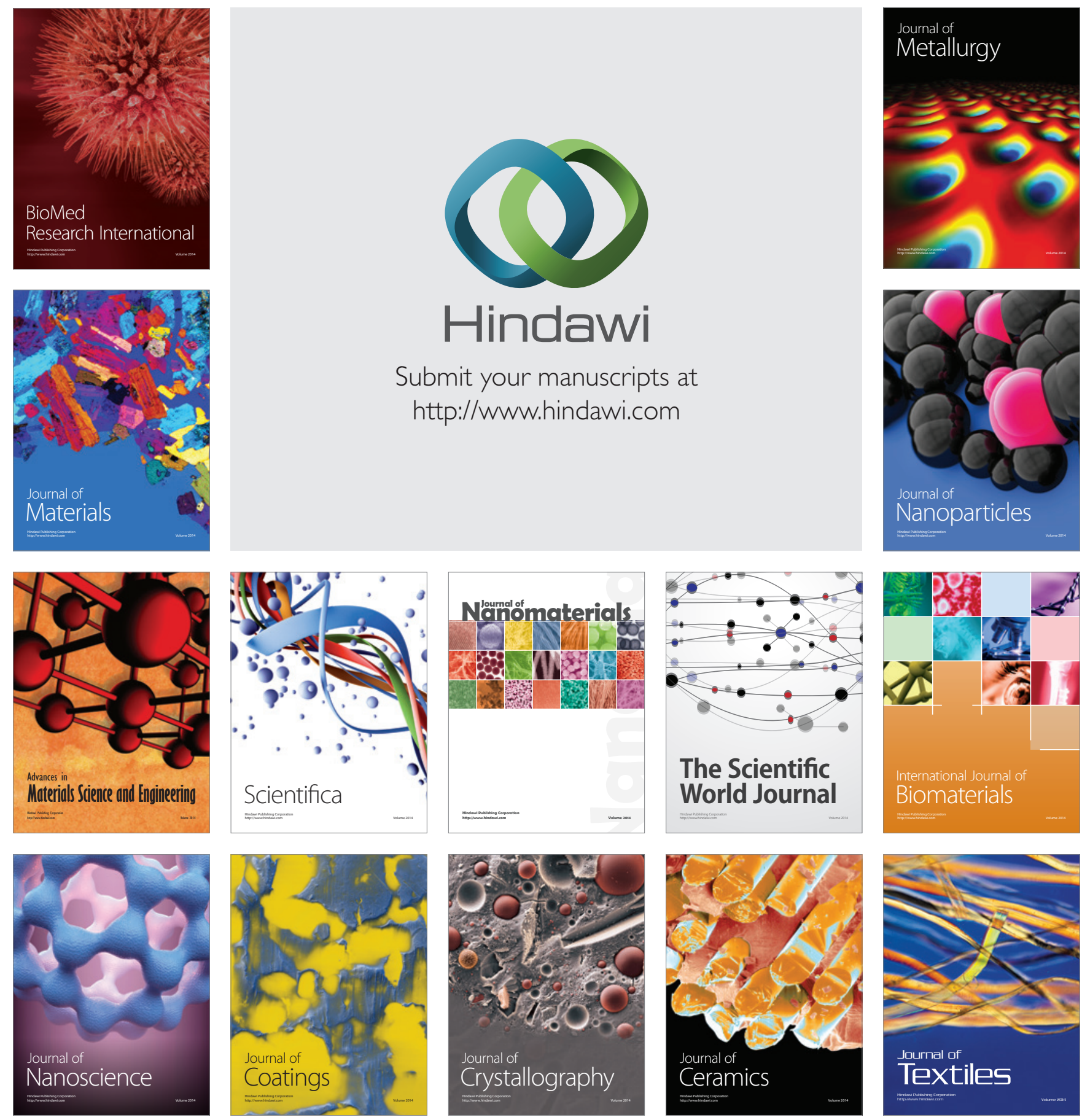